\title{
Business Report: Is the Shared Fascia Gun Plausible in China?
}

\author{
Haopeng Bai
}

Tieyi high school,Xi'an, 710054, China,1561355221@qq.com

\begin{abstract}
After exercise, people usually seek for a way for relaxing muscles. Otherwise, their muscles and joints will become stiff. If this situation continues for a long time, it will cause great damage to people's body. And the fascia gun is a convenient and inexpensive way to effectively relieve muscle pressure. Under the shared economy model in China, there are more and more products combine with the idea of sharing to produce new products that benefit mankind. The objective of this work is to evaluate the plausibility of putting shared fascia gun in some specific areas in China.
\end{abstract}

Keywords: Shared fascia gun, innovation, Market orientation, plausibility

\section{INTRODUCTION}

The product that showing in figure 1 is a combination of fascia gun and a vending machine, it can allow people to use it in an easy and inexpensive way which is an effective approach to get a place in the new market.

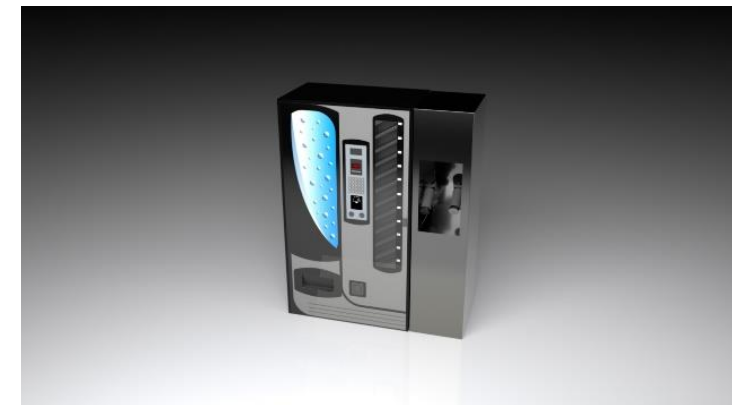

Figure 1 Shared fascia gun design concept drawing

\section{BACKGROUND}

\subsection{What is the fascia gun?}

After exercise, muscles will tense, lactic acid accumulation, lack of oxygen, especially after excessive exercise, muscles are very stiff, difficult to recover on their own. The outer layer of human muscle will have a layer of fascia wrapping, so that muscle fibers can produce contraction in an orderly direction, to achieve a better functional state [1]. After excessive exercise, the muscle fascia will be inflated or squeezed, resulting in pain and discomfort [2]. The fascia gun solves muscle tension and other problems through external physical means, which is similar to the function of ligament stretching, muscle pulling and auxiliary manual massage used by professional athletes after finishing training [3]. The fascia gun uses the special high-speed motor inside to drive the "spear head", and generates high-frequency vibration to the deep muscle to reduce the tension of local tissue, relieve pain and promote blood circulation [4]. With the help of the fascia gun, the recovery time and efficiency after exercise will be improved. Properly and properly used, the fascia gun can indeed help relieve muscle soreness after exercise [5].

\subsection{How to use the fascia gun?}

Whether you're after a certain spot on the body or an end-of-day release for your whole system, you should use the fascia gun in five steps.

1. Turn on the fascia gun without putting it on your body. That may seem like a minor thing, but it's important for better control. And resting the fascia gun on your muscle and then turning it on can be a little jarring.

2. Rest the fascia gun on your body. Don't add any pressure. Just let it float.

3. Glide the fascia gun along the muscle. Move slowly-about an inch a second or slower.

4. If you hit a knot or an area of tension, let the fascia gun rest on that spot-without adding additional pressure - for a few moments before moving on.

5. Breathe: nice long inhales and exhales. And feel the relief [6]. 


\subsection{The marketing history in US and China}

Before Hyperice was founded, Anthony Katz was a high school teacher in California. He began studying sports rehabilitation because of his love of basketball, which often led to many physical injuries.

So he founded Hyperice company and begin to manufacture the fascia gun, which was launched in the United States in 2017 and introduced to China in late 2017 [7].

With more and more people using fascia gun in the past two years, and with the support of domestic and foreign stars, this massager has become one of the indispensable tools for any sports, fitness and even home rehabilitation.

In recent years, with the rapid development of China's economy, people's demand for a healthy life has increased significantly, the awareness of proper massaging after exercise has been enhanced and thus the potential market of fascia gun is expanding accordingly. Data shows that the sales volume of fascia gun increased by $2,177 \%$ in $19-20$ years. While advocating national fitness, Chinese government also made favorable plans for the fascia gun industry. It clearly suggested that the fascia gun industry in China would thrive [8].

\section{PLANNED BUSINESS MODE}

In order to popularize fascia gun in China, I finally came up with this idea after some research. Unlike the passion and craziness towards sport stars in U.S., the love of sport has been exhibited in a less passionate manner in China.

During the 2017 NBA Finals, Cavaliers guard Kyrie Irving quickly returned to the court using a fascia gun to relax his back and thigh muscles with the help of a physical therapist [9].

Thus, sports stars in China cannot be so influential. Besides, as a developing country, most Chinese cannot accept a price of almost 800 dollars for a device of pure physical relaxation. As a result, my plan is to create a product that could combine the functions of a fascia gun and a vending machine. Consequently, people can use fascia gun by paying a small amount through coins or OR code, so people do not need to spend a lot to buy one fascia gun and still have access to using it at the end of the exercise to recover quickly and efficiently.

\section{PROCEDURES TO TEST ITS PLAUSIBILITY}

I divided my research into two parts, one is to keep track of the percentage of people who stretch before or after exercise and other relevant details, and the other is to identify the advantages and disadvantages of my design.

\subsection{Getting to know the potential customers}

I got the feedback from potential customers through a survey.

My survey questions are all multiple choice questions. Some of the key questions are as follows:

1.How much do you know about fascia guns?

A. never heard about it B. heard about it but do not know any details

C. used it once time D. sometimes use it E. almost use it almost everyday

2. Do you stretch after exercise, and if so, have you ever used a fascia gun?
A. No, No
B. Yes, No

C. Yes, Yes

3. under the same charge upon each use, which one would you prefer?

A. a cheap, disposable, replaceable massage head

B. a high-quality, reusable massage head that should be replaced once a week and disinfected after each user

4. Which of the following prices do you think is the reasonable price for each use (10 minutes each time)?
A. $0-5$
B. $5-10$
C. $10-15$
D. $15-20$

5. How much do you generally spend on the exercise (include any sports) every year?
A. $0-500$
B. $500-1500$
C. $1500-2500$
D. $2500-4000$
E. more than 4000

Considering that the data collected from random people is not of great reference significance, I narrowed the scope of the survey population and only conducted the survey in the gym, basketball hall and the football and basketball club of the school.

\subsection{Survey results in brief}

\section{How much do you know about fascia guns?}

I ended up surveying 60 people, and according to the result of this question, 48 of them had heard of a fascia gun, but 36 of them had never used one.

2. Do you stretch after exercise, and if so, have you ever used a fascia gun?

For the second question, $60 \%$ students obviously had some misunderstandings about the fascia gun, and these people had the habit of relaxing after exercise but had never used the fascia gun to help them. 
3. Under the same charge upon each use, which one would you prefer?

In response to the third question, what surprises me is that over $60 \%$ of people chose disposable replaceable massage heads.

4. Which of the following prices do you think is the reasonable price for each use (10 minutes each time)?

The majority think the most suitable price is 5-10 yuan for each use. In addition, according to the general preference of the disposable massage head (question No. 3 ), I plan to include the disposable gun massage heads for sale in the design. So people can choose to use the regularly-disinfected massage head for free or can buy the cheap gun massage heads from our machine if they are really concerned about sanitary risks or just uncomfortable to share things with other people.

\section{MARKETING STRATEGIES}

Product promotion is an essential part, and my marketing strategy has the following two schemes.

1. I cooperate with fitness clubs, basketball courts and other similar places with exercise habits and share the profits. I provide the machine and maintain it regularly, while the partner pays the electricity and advertises it to its members, and $10 \%$ of the profits go to the partner.

The advantage of doing this is that my advertising cost can be greatly reduced. Besides, the gym and the basketball arena can independently manage my machine and report the maintenance when the machine breaks down or needs to be repaired, which can also improve the use efficiency of the machine. At the same time, driven by profit, the partner organizations themselves will take advantage of corresponding publicity means, such as giving coaches free trial opportunities, so that they can convince their members to use the machine with their personal experience. In addition, an annual member of a gym could enjoy the use of a free fascia gun after each workout. All of this helps to boost my machine and increase consumer demand.

However, the scheme also has its own disadvantages. Placing a machine in a organization means that my profits will be largely in the hands of others, and if the partner organizations are not properly managed or advertised then the profits will be out of proportion to my investment. In addition, we should spend a lot of money on maintenance and replacement, so if the partner organization does not pay attention to the maintenance of the machine, then these extra costs will continue to grow.

2. I sign a contract with the partner organization where the fascia gun machine is placed and the partner provides me space to place my own machine and also take full responsibility of regular maintenance and publicizing among their members. The advantage is that many unnecessary damage to the machine can be avoided; the machine can be in a good condition for the normal use. Their members are target clients so publicizing among them is highly efficient. However, the disadvantage of doing this is also obvious. That is, I have to give a lot of money to partners for publicity and maintenance, and my cost will dramatically increase.

But in either case, I do the outside publicity myself and the publicity of this part is as follows:

1. I will set up an independent public account about shared fascia guns, and update articles about the proper use and the benefits of fascia guns.

2. I will print leaflets about my machine and distribute them in the vicinity of gyms and places alike. On the leaflets, there will also be the QR code of my official account and the location nearby, so that people can quickly learn about my product and try it.

3. The QR code of my official account will be printed on the machine. Before any potential customers use the fascia gun, a notice will be clearly on the machine that they can have a free trial for the first time by following the official account, and if they purchase next time, they will be able to use it at half the price for three more times.

In order to make effective moves as above, I also consulted a professor from Yan 'an University to understand the mode of advertising and got some suggestions: advertisements should be placed in health clubs, public sports venues, traffic stations, airports and railway stations, where people have a lot of time and are willing to stop for new information. At this time, they are more likely to pay attention to advertising.

\section{COST EVALUATION}

For cost, in order to know whether my plan can be carried out, in the trial phase I plan to put only four machines in four gyms for a quarter of the year. Each machine includes vending massage head fascia gun, vending machine fascia gun wrap, and each fascia gun costs about 200 yuan, if each machine equipped with nine fascia guns, it will cost about 10,000 yuan, so the total cost of four machines is 40,000 yuan [10]. In addition to the cost of the machine, there are also advertising costs and cost based on different partnership pattern. So I divided my next cost into two categories based on my marketing strategy.

The first one is to cooperate with the gym, so I need to put the posters of shared fascia gun machine in the elevator of the gym. The cost of this advertisement is 900 yuan/quarter, and if the gym has two elevators, the total cost of four gyms will be 7,200 yuan [11]. Besides, I need to hire 4 people to send out flyers on the streets near the gym for a week. This will need roughly 2800 leaflets. 100 leaflets cost 35 yuan (including leaflet design. 80 yuan a 
day is required to hire one person a day, and a week is 560 yuan [12]. Totally, the cost of my advertising distribution outside is 600 yuan, the total cost is 7800 yuan, plus the cost of the machine is 47,800 yuan.

The second pattern: Since I sign a contract with the gym, I plan to give 20,000 yuan to the gym in a quarter, and accordingly, the gym needs to publish an article about sharing fascia gun machine entering into the gym on its official account and put up posters in the gym. If customers have needs, they should also actively respond to customers. At the same time, when the machine is out of order, it should be repaired and reported immediately. If a client uses a fascia gun at the end of the workout, someone in the facility who is free will need to help. The total cost of this scheme is 60,000 yuan.

\section{OTHER CONCERNS}

Despite my rigorous and thorough analysis, there are still some problems that I find difficult to control.

1. Will the trial phase recoup costs or even make money, and if not what will be the next? Another threemonth trial or just check the conditions every single day?

I have some figures here, but the decision still remains unclear.

In the trial phase, according to the usage times of 20 people per gym every day, there will be 80 person-times per day, and there will be about 7,000 people in a quarter. Under my current ideal price, it is 5 yuan to rent a gym for 10 minutes and 5 yuan to buy a disposable massage head, so the use price of each time is 10 yuan. The profit at the end of the trial phase is 70,000 yuan, so I can get my money back in three months. But this is the ideal state. If It doesn't reach the desired result after the trial period, I do not know which is more practical.

2. Will the promotion activities in the early stage make the customers get used to the discount, and will the customers stop using the fascia gun once the price gets back to standard?

\section{CONCLUSION}

After the above analysis, I think this product still has a certain prospect and market, so I plan to conduct a trial operation for one quarter first to see the results. After all, no one can guarantee that a business plan will never fail and be free of trouble along the way.

In the work, I through to the fascia gun background check and social research and the success of the understand the feasibility of sharing fascia gun in the Chinese market, also the cost estimates in the development of the Chinese market and work out a plan for the first quarter of the trial operation, it can be seen that the Shared fascia gun will share to the success of economic and professional fitness equipment together and gave birth to a viable new products in the Chinese market, There will definitely be a place for shared fascia guns in the future Chinese market.

\section{REFERENCES}

[1] Cleveland Clinic-What is myofascial pain syndrome? https://my.clevelandclinic.org/health/diseases/120 54-myofascial-pain-syndrome

[2] William Gibson 2009 Jan 21 Increased pain from muscle fascia following eccentric exercise: animal and human findings

https://pubmed.ncbi.nlm.nih.gov/19156402/

[3] Karen Asp July 1, 2021 Everything You Need to Know About Massage Guns

https://www.everydayhealth.com/fitness/everythin g-you-need-to-know-about-massage-guns/

[4] dabaitunaitang00 2021-04-27 What is the principle of fascia gun?

https://zhidao.baidu.com/question/171069495708 9637940.html

[5] Alitarui Trading December 5th, 2020 Are there any side effects of the fascia gun?

https://www.alitarui.com/2020/12/05/are-thereany-side-effects-of-the-fascia-gun/

[6] Goop- how to use theragun in five steps

https:/goop.com/wellness/fitness/how-to-usetheragun/

[7] Tengxun Wang June $19^{\text {th }}, 2019$ The origin of the fascia gun -HYPERICE https://zj.qq.com/a/20190619/002180.htm

[8] Yunshe Sui September $16^{\text {th }}, 2020$ Overview of fascia gun industry and market analysis https://www.jianshu.com/p/3acec9517c3d

[9] Cang Liang March $8^{\text {th }}, 2020$ The fascia gun used by celebrities and fitness fanatics

https://www.sohu.com/a/299880765_162067

[10] Walmart-Massage Gun

https:/www.walmart.com/ip/Fascia-Gun-HandHeld-Deep-Tissue-Muscle-Massage-GunPortable-Cordless-Percussion-Vibration-FasciaMassager-Device/502698310

[11] Doodian-Advertisment

https://www.doodian.com/news/fenzhong/2021/0 319/721.html?bd_vid=7000724871869950301

[12] Beijing Yingxin cultural advertising company http://www.xinyingxin.cn/col.jsp?id=103 